\title{
Noncontrast Magnetic Resonance Lymphography
}

\author{
Lionel Arrivé, $\mathrm{MD}^{1} \quad$ Sarah Derhy, $\mathrm{MD}^{1} \quad$ Sanaâ El Mouhadi, MD ${ }^{1} \quad$ Laurence Monnier-Cholley, MD ${ }^{1}$ \\ Yves Menu, MD ${ }^{1}$ Corinne Becker, MD $^{2}$
}

${ }^{1}$ Department of Radiology, AP-HP Groupe Hospitalier Saint-Antoine,

Address for correspondence Lionel Arrivé, MD, AP-HP Groupe

Paris, France

2 Department of Surgery, AP-HP Hôpital Européen Georges Pompidou, Hospitalier, Saint-Antoine, 184, rue du Faubourg Saint-Antoine, 75571

Paris, France (e-mail: lionel.arrive@sat.aphp.fr).

Paris, France

J Reconstr Microsurg 2016;32:80-86.

\begin{abstract}
Background Different imaging techniques have been used for the investigation of the lymphatic channels and lymph glands. Noncontrast magnetic resonance (MR) lymphography has significant advantages in comparison with other imaging modalities.

Methods Noncontrast MR lymphography uses very heavily T2-weighted fast spin echo sequences which obtain a nearly complete signal loss in tissue background and specific display of lymphatic vessels with a long T2 relaxation time. The raw data can be processed with different algorithms such as maximum intensity projection algorithm to obtain an anatomic representation.

Results Standard T2-weighted MR images easily demonstrate the location of edema. It appears as subcutaneous infiltration of soft tissue with a classical honeycomb pattern. True collection around the muscular area may be demonstrated in case of severe lymphedema. Lymph nodes may be normal in size, number, and signal intensity; in other cases, lymph nodes may be smaller in size or number of lymph nodes may be restricted. MR lymphography allows a classification of lymphedema in aplasia (no collecting vessels demonstrated); hypoplasia (a small number of lymphatic vessels), and numerical hyperplasia or hyperplasia (with an increased number of lymphatic vessels of greater

Keywords

- lymphatics

- lymphedema

- MR imaging

- MR lymphography and abnormal diameter).

Conclusion Noncontrast MR lymphography is a unique noninvasive imaging modality for the diagnosis of lymphedema. It can be used for positive diagnosis, differential diagnosis, and specific evaluation of lymphedema severity. It may also be used for follow-up evaluation after treatment.
\end{abstract}

The primary lymphedema affects the lower limb more frequently than the upper limb. Lymphedema of the lower limb is mainly characterized by tissue swelling which may be present at birth or appears at different ages throughout life. Primary upper limb lymphedema is markedly uncommon. On the contrary, secondary lymphedema of the upper limb is a common disease usually related to a complication of breast carcinoma and its treatment including surgery and radiation therapy.

received

December 17, 2014

accepted

February 1, 2015

published online

March 31, 2015

\section{Imaging Modalities of Lymphatic Diseases}

Although advanced forms of lymphedema present characteristic clinical features resulting from changes in the skin and subcutaneous tissues, early-stage manifestations may be restricted to swelling which may be present in other diseases. ${ }^{1}$ Therefore, investigation of lymphedema may be useful to confirm the diagnosis, to evaluate the severity of lymphedema, to perform the differential diagnosis, and to allow a

Copyright $\odot 2016$ by Thieme Medical Publishers, Inc., 333 Seventh Avenue, New York, NY 10001, USA. Tel: +1(212) 584-4662.
DOI http://dx.doi.org/ $10.1055 / \mathrm{s}-0035-1549133$. ISSN 0743-684X. 
classification of lymphedema. In addition, different techniques may be used during follow-up after treatment. ${ }^{2}$

Different imaging techniques have been used for the investigation of the lymphatic channels and lymph glands.

For a long time, X-ray lymphography, the so-called direct lymphography, has been the single method to explore the lymphatic system. ${ }^{3}$ Presently, X-ray lymphography is no more used for cancer assessment. However, it is still performed for the study of lymphatic circulation disorders in selected indication. Several contraindications must be respected: cardiovascular failure, lung infection, respiratory failure, and cardiac right-left shunt. X-ray lymphography is performed by injecting a radio-opaque material into a lymphatic that has been surgically exposed and cannulated. ${ }^{4}$ After cannulation, the catheter is connected to a syringe to perform the injection of lipiodol ultrafluid which allows opacification of lymphatic channels and lymph nodes. Allergic reaction may occur after injection, pneumonitis, and pulmonary embolism have been described. The lymphatic vessels are opacified during the first day after injection; they appear as regular channels in the lower limb, the inguinal and retroperitoneal areas. They continue through the thoracic duct into the venous confluent between internal jugular vein and subclavian vein. Lymph nodes may be analyzed during the second day; they appear as oblong formations with a granular component extending from sinus node to the periphery. One observes large variations in number and size of lymph nodes. However, X-ray lymphography is a rather long and invasive procedure which is very uncommonly performed in special selected indication.

Computed tomography (CT) is more currently available. It may be used for the direct diagnosis of upper or lower limb lymphedema. CT is able to provide information related to the volume change of lower or upper limb. It can also demonstrate the honeycomb pattern commonly observed in case of lymphedema. ${ }^{5}$ In addition, a CT scan may be performed the first day of classical X-ray lymphography. It is the so-called lympho-scanner. This helical CT performed with multidetector scanner can demonstrate the lymphatic vessels in abdominal and thoracic areas. It is an interesting imaging modality mainly performed in selected indications to demonstrate lymphatic leaks.

Ultrasonography is marginally used for the investigation of lymphedema to analyze the distribution of fluid within the different parts of the limb. ${ }^{6}$ Similarly, the upper end of thoracic duct may be observed by means of ultrasonography.

Lymphoscintigraphy can provide indirect exploration of the lymphatic system by means of analysis of drainage of radiolabeled particles injected into the subcutaneous tissue, in contrast to the direct injection of lipiodol within the lymphatic lumen during X-ray lymphography. Labeled tracer is a suspension of nanocolloid labeled with technetium-99m. It takes advantages of the essential capability of the lymphatic to clear large molecules from the interstitium. Scintigraphy using a gamma camera provides an assessment of the tracer distribution in the leg and its uptake through the lymph nodes. $^{7}$

Lymphoscintigraphy is a minimally invasive technique which is currently performed as a usual screening technique in case of clinical diagnosis of lymphedema. The injection of labeled tracer is bilateral within the subcutaneous tissue of the first interdigital space of the hands or feet. Imaging protocols are not standardized and depend on institution habits. Sequential images of the progression of the labeled tracer are recorded by a gamma camera. After subcutaneous injection, diffusion of the labeled tracer is observed into lymphatic capillaries with accumulation in lymph nodes. Lymphoscintigraphy can be used to eliminate other causes of edema. It also provides insight into lymph flow dynamics, participates to the therapeutic decision, and can be repeated during the follow-up.

\section{Lymphatic Magnetic Resonance Imaging}

Magnetic resonance (MR) imaging provides a unique highquality contrast between different soft tissues. In addition, it provides a superb contrast between water, fat, and other soft tissues. ${ }^{8}$ It is a noninvasive imaging modality without use of $\mathrm{X}$-rays. Contraindications are restricted to severe claustrophobia, pace makers, and ferromagnetic intracerebral clips. For the evaluation of lymphatic system, MR imaging may be performed with specific superparamagnetic contrast media, with subcutaneous injection of paramagnetic contrast media or without any contrast media injection, it is the so-called noncontrast MR lymphography. For many years, conventional MR imaging has been used to diagnose metastases in lymph nodes. Conventional MR imaging relies on the enlarged size of lymph nodes. However, there are many noncancerous causes of lymph node enlargement that can result in false-positive diagnosis. In addition, very small metastases can remain undetected.

\section{Ultra-Small Super-paramagnetic Iron Oxide Nanoparticles-Enhanced MR Imaging}

The principles of ultra-small super-paramagnetic iron oxide (USPIO)-enhanced MR imaging relies in intravenous injection of these ultra-small particles. The particles are taken up by the lymphatic and transported to the lymph nodes. It results in enhancement of the normal lymph nodes which become dark on T2-weighted MR imaging, while lymph nodes metastases remain unchanged. ${ }^{9}$ This technique is currently used for the diagnosis of lymph nodes metastases. On the contrary, USPIO-enhanced MR imaging is not suitable for lymphatic channels imaging and for the diagnosis of lymphedema.

\section{Contrast-Enhanced MR Lymphangiography}

The principle of contrast-enhanced MR lymphangiography is to subcutaneously inject paramagnetic macromolecules larger than 6 to $10 \mathrm{~nm}$ that preferentially enter the lymphatic capillaries and are retained throughout the length of the lymphatic channels resulting in contrast enhancement within lymphatic vessels and lymph nodes. Therefore, contrastenhanced MR lymphangiography allows the visualization of lymphatic drainage. ${ }^{10}$ The size of contrast media macromolecules is critical for its efficient uptake by the lymphatic 
vessels. Contrast-enhanced MR lymphangiography demonstrates several advantages over lymphoscintigraphy. The contrast/noise ratio is high and the spatial resolution is far better than lymphoscintigraphy. Contrast-enhanced MR lymphangiography can be used to precisely locate the lymphatic channels and the lymph nodes. Enhancement of lymphatic channels is of excellent quality at the proximity of contrast media injection site. On the contrary, visualization of more distal lymphatic vessels may be more difficult.

\section{Noncontrast Magnetic Resonance Lymphography}

Whole-body MR imaging has significant advantages in comparison with other imaging modalities. Main advantages include very high soft tissue contrast (especially for water), multiplanar capability, and no need for ionizing radiation or contrast material. Initially, limitations were mainly related to long image acquisition time that resulted in image degradation due to various biologic motions. This limitation is now overcome by technical advances in different areas: gradient performance, pulse sequence, and coil improvement. Improvement of gradient performance allows faster rise times and improved slew rates and therefore allows a dramatic reduction in data acquisition. New pulse sequences can be used with faster data acquisition including a greater number of sections, thinner sections, and acquisition of isotropic dataset. Introduction of new sequences such as fast spin echo or turbo spin echo allows the generation of a train of echoes allowing K-space filling in one shot. Fast spin echo has been used in T2-weighted imaging allowing acquisition of spin echo sequences in a few minutes and single shot fast spin echo sequences in a few seconds. Indeed, these sequences allow obtention of images with specific target on static fluid within organs tube or spaces. Therefore, sequences of MR cholangiopancreatography, MR urography, and MR myelography are currently performed routinely. ${ }^{11}$ The principle of these sequences is to use very heavily $\mathrm{T} 2$-weighted fast spin echo sequences with a very long echo train so that the effective echo times are greater than the tissue relaxation time. Therefore, one obtains a nearly complete signal loss in tissue background with specific display of static fluids with a long T2 relaxation time. Therefore, bile, urine, or cerebrospinal fluid appears as white structure on a black background without need of any injection of contrast media. The raw data can be processed with different algorithms such as maximum intensity projection (MIP) algorithm to obtain an anatomic representation. Initially, bi-dimensional sequences were used; however, three-dimensional (3D) acquisitions allow exploiting the multiplanar capability of MR imaging. With further improvements in both hardware and software equipments, imaging of smaller structures is now more commonly feasible. However, because MR lymphography is a relatively new imaging technique, and because of the ongoing advances in software and hardware, the technique is still evolving. We commonly use a free-breathing 3D high-spatial-resolution fast spin echo sequence with an extremely long TE. A -90degree radiofrequency pulse (the so-called restore pulse) is applied at the end of the echo train to flip the transverse magnetization to the longitudinal direction to shorten the spin relaxation time and accelerate the relaxation of the longitudinal magnetization while maintaining the same contrast resolution and reducing the acquisition time. In most cases, we use a symmetric matrix of $256 \times 256$, the section thickness is $1 \mathrm{~mm}$ and the voxel size is $1 \times 1 \times 1 \mathrm{~mm}$. In selected cases, we used $512 \times 512$ matrix and section thickness inferior to $1 \mathrm{~mm}$. Postprocessing of the image data is performed to obtain MIP images and multiplanar reformatted images. The typical acquisition time is 3 to 6 minutes for respiratory-triggered navigator-gated acquisition with a prospective acquisition correction PACE technique. ${ }^{12}$

$3 \mathrm{D}$ imaging technique is performed in all cases because it has definitive advantages over $2 \mathrm{D}$ imaging technique including the capacity to obtain thinner section with no gap and higher signal-to-noise ratio. Images are reconstructed with thin, thick, and volume MIP reconstructions. To analyze the abdomen or pelvis, patient should drink $500 \mathrm{~mL}$ of pineapple juice (characterized by a very high concentration of manganese) to decrease signal intensity of fluid present within small bowel. ${ }^{13}$ The bladder should be empty at the beginning of MR examination.

Multiple channels phased array coils are adapted to the specific field of view. In specific indication, surface coils may be used. With such protocol, inguinal lymphatic vessels, retroperitoneal lymphatic vessels, and thoracic duct are always well demonstrated. ${ }^{14}$ Main vessels of lower limbs are usually demonstrated. On the contrary, normal lymphatic vessels of upper limbs are less easily analyzed. We evaluate lymph nodes with a combination of $\mathrm{T} 2$-weighted fast spin echo sequences including fat-suppressed, in and out of phase sequences, and in specific indications diffusion-weighted MR sequences are also used. Use of high field MR imaging will potentially improve the signal-to-noise ratio. However, extensive experience of such results is still missing.

\section{Anatomy}

The lymphatic system is in close relation to the blood vascular system. It is composed of vessels that drain interstitial fluid that returns into the venous circulation. These lymphatic vessels pass through lymph nodes containing lymphoid tissue. Therefore, lymphatic system plays a vital role against infectious diseases and cancer. The lymphatic system is difficult to demonstrate.

\section{Thoracic Duct}

The main lymphatic vessel is the thoracic duct. Classically, the thoracic duct is formed by the union of two retroperitoneal lymphatic trunks and a single mesenteric lymphatic trunk at the level of the cisterna chyli. However, the abdominal and retroperitoneal lymphatic system is characterized by a lot of anatomic variations. Appearance of retroperitoneal lymphatic vessels varies from thin to prominent thick channels, parallel or converging channels or plexuses. The cisterna chyli receives right and left retroperitoneal lymphatic trunks and 
the intestinal lymphatic trunk. The cisterna chyli is usually described in anatomic books as a saccular area of dilatation of the lymphatic channel at the L1-L2 level of the vertebral body. However, in most cases, the cisterna chyli has a highly variable appearance. ${ }^{12}$ Therefore, several authors prefer the descriptive term "abdominal confluence of the lymphatic trunks." ${ }^{8,10,12}$ Thoracic duct terminates at the junction of the left subclavian and internal jugular veins draining lymph into the venous circulation. However, thoracic duct is also characterized by numerous anatomic variations. At its origin, it is very common to observe two or three trunks or a plexus of converging channels. Such plexus can also be observed at the middle of the thorax. Right thoracic duct or double right and left thoracic duct within the thorax are less common.

\section{Lymphatic Vessels}

The lymphatic vessels are of three types: initial lymphatic vessels, precollector, and collector lymphatic vessels. Collector vessels can only be demonstrated with noncontrast MR lymphography. Both precollector and collector lymphatic vessels present with valves which prevent backflow of lymph. Between two sets of valves is a contractile unit of smooth muscle that allows the progression of lymph. Lymphatic vessels are demonstrated with MR lymphography as alternating areas of constriction and dilatation representing valves and contractile units.

\section{Lymph Nodes}

With MR lymphography, lymph nodes of the lower limbs are uncommonly demonstrated. The more commonly demonstrated is the popliteal node within the fat of the popliteal fossa. The inguinal lymph nodes are easily demonstrated at the level of the inguinal ligament. They are markedly variable in size and number. Retroperitoneal lymph nodes are also easily demonstrated. At the upper limbs, lymph nodes are uncommonly demonstrated. On the contrary, axillary lymph nodes are easily seen. Whatever their localization, MR appearance of normal lymph nodes is markedly variable. Some nodes appear as a converging plexus of small lymphatic channels. In other cases, a large fatty component is present in the center of the lymph nodes. Therefore, signal intensity of lymph nodes may be markedly variable.

\section{Lymphedema of the Limbs}

Standard T2-weighted MR images easily demonstrate the location of edema. It appears as a subcutaneous infiltration of soft tissue with a classical honeycomb pattern. True collection around the muscular area may be demonstrated in case of severe lymphedema (-Fig. 1). Thickening of the dermal plane is frequently demonstrated in chronic lymphedema. On the other hand, the subfacial (muscular) compartment of the swollen limb demonstrates absence of muscle edema, thereby confirming that lymphedema is primarily a disorder of subcutaneous tissue (-Fig. 1). Therefore, it is easy to differentiate lymphedema from venous edema with involvement of the muscular compartment of the swollen limb. It is also easy to differentiate lymphedema from lipedema; in the latter case, limb enlargement is only related
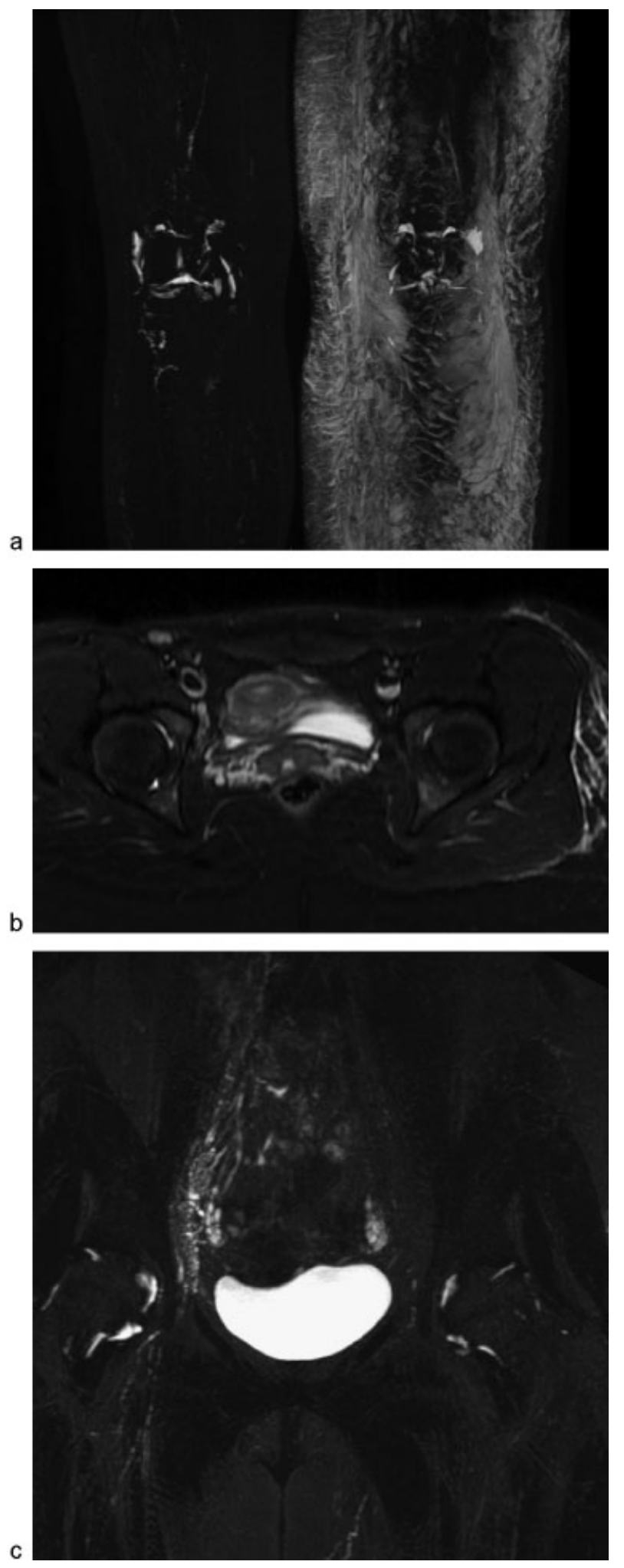

Fig. 1 Hypoplasic left lower limb lymphedema in a 23-year-old female. MR lymphography at the knee level (a) demonstrates an infiltration of the subcutaneous tissue with true collection around the muscular area. Inguinal nodes are absent on the left side (b) and hypoplasia of both left iliac and inguinal lymphatic vessels are demonstrated (c). 

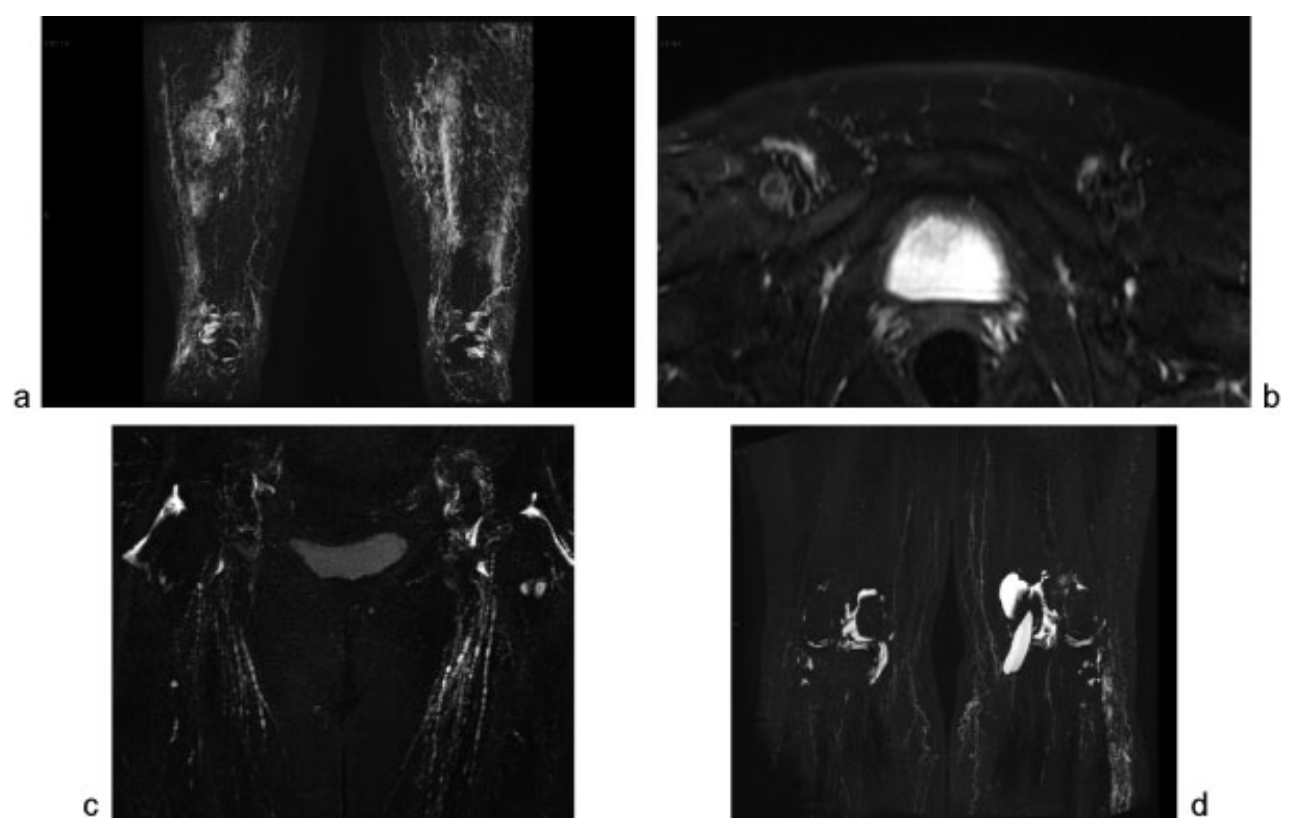

Fig. 2 Bilateral hyperplasic lymphedema of lower limbs. Subcutaneous infiltration is demonstrated on both limbs (a). Hyperplasia of small lymphatic vessels is well demonstrated. Normal inguinal nodes are well demonstrated (b). Hyperplasia of inguinal lymphatic vessels (c) and lymphatic channels of both legs (d) are well seen.

to fat deposition rather than to fluid accumulation. However, lipedema may be associated with lymphedema, as accumulation of protein-rich fluid in the tissues causes overgrowth of adipose tissue. In this case, MR lymphography easily demonstrates combination of fat deposition with fluid accumulation. In addition, MR lymphography is a unique imaging modality to demonstrate contralateral minimal or moderate lower limb lymphedema not visible with clinical examination.

\section{Lymph Nodes}

Analysis of inguinal lymph nodes is easily performed by means of noncontrast MR lymphography. Lymph nodes may be normal in size, number, and signal intensity in case of lower limb lymphedema (-Fig. 2). In other cases, lymph nodes may be smaller in size or number of lymph nodes may be restricted and/or signal intensity may be altered (-Fig. 1 ).

\section{Analysis of Lymphatic Vessels}

The original classification of primary lymphedema by Kinmonth et $\mathrm{al}^{3}$ was based on the lymphographic patterns obtained with X-ray lymphography. Lower limbs lymphedemas were classified as follows:

- Aplasia: no collecting vessels are demonstrated

- Hypoplasia: a small number of lymphatic vessels are visible (-Fig. 1)

- Numerical hyperplasia: more normal size vessels are demonstrated

- Hyperplasia: an increase number of lymphatic vessels of greater and abnormal diameters are demonstrated (-Fig. 2)
Noncontrast MR lymphography allows such classification. However, lymphatic vessels analysis should be combined with lymph nodes analysis. In addition, several lower limb lymphedemas may be observed with a normal appearance of both lymph nodes and lymphatic vessels.

Finally, in uncommon cases, diffuse abnormalities of lymphatic vessels may be observed with the appearance of diffuse lymphatic dysplasia. In case of secondary lower limb lymphedema occurring after obstruction of lymphatic vessels related to surgery and/or radiation therapy, it is important to analyze both lymph nodes and lymphatic vessels. In most cases, a fewer number of lymph nodes are demonstrated. Interruption of lymphatic vessels with or without distal lymphatic vessels dilatation may be observed (-Fig. 3 ).

\section{Follow-Up Evaluation}

After treatment, noncontrast MR lymphography allows a precise evaluation of lymphedema. Volume of affected lower limbs may easily be measured. In addition, severity of infiltration of subcutaneous tissue and volume of collection of free fluid outside the muscular compartment may easily be evaluated.

After lymph nodes autotransplantation, the transplanted lymph nodes may be directly demonstrated. On the other hand, lymphovenous anastomosis is commonly not demonstrated and analysis of lymphatic neovessels is extremely difficult.

\section{Conclusion}

Noncontrast MR lymphography is a unique noninvasive imaging modality for the diagnosis of lymphedema. 


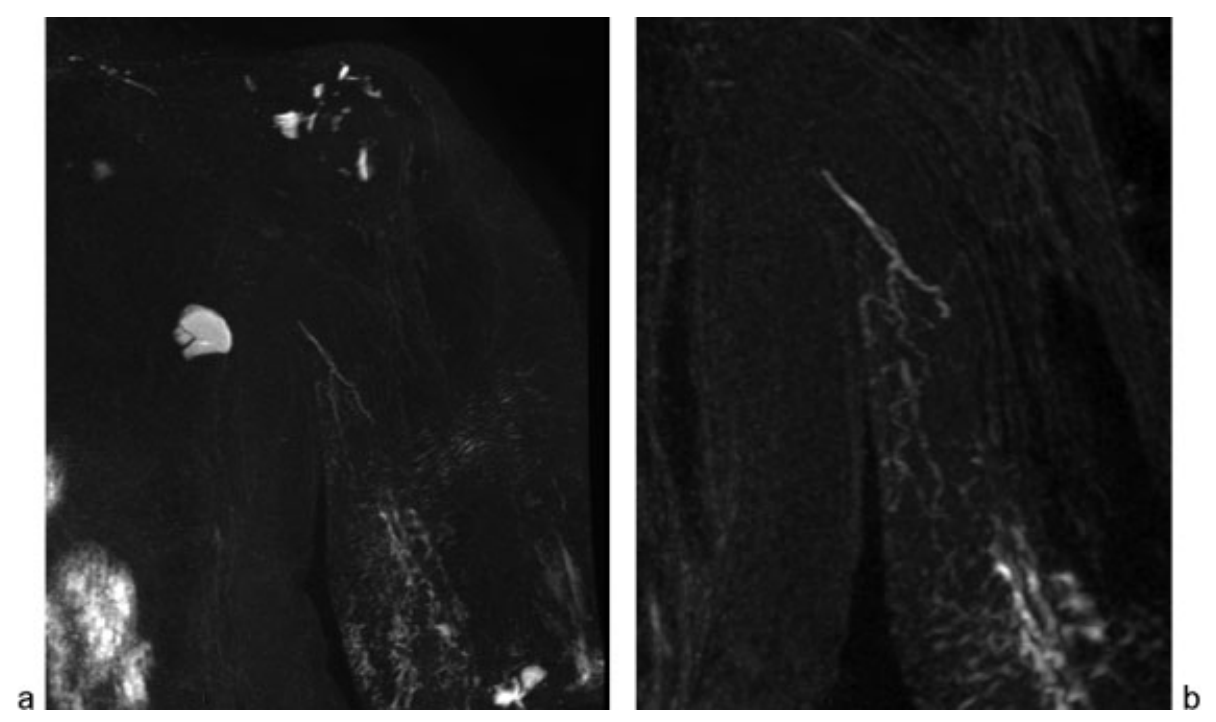

Fig. 3 Secondary lymphedema of left upper limb in a 64-year-old female after surgery and radiation therapy for breast carcinoma. A lymphedema is demonstrated as an infiltration of subcutaneous tissue (a), and dilatation and interruption of lymphatic vessels are well demonstrated (b).

We believe that noncontrast MR lymphography is important for positive diagnosis, differential diagnosis, and specific evaluation of lymphedema severity. It may also be used for follow-up evaluation after treatment. Finally, it allows specific classification of lymphedema with combination of both lymphatic vessels and lymph nodes abnormalities.

However, noncontrast MR lymphography is a relative new imaging technique. It should be considered as a work in progress imaging modality. The main limitation today is the suboptimal spatial resolution. However, because of ongoing advances in software and hardware, the technique is evolving, and we believe that in near future, it could become the imaging modality of reference for investigation of lower limb lymphedema, upper limb lymphedema, and other lymphatic abnormalities.

\section{References}

1 Browse N, Burnaud KG, Mortimer PS. Diseases of the lymphatics. Arnold, London; 2003

2 Witte CL, Witte MH, Unger EC, et al. Advances in imaging of lymph flow disorders. Radiographics 2000;20(6):1697-1719

3 Kinmonth JB, Taylor GW, Tracy GD, Marsh JD. Primary lymphoedema; clinical and lymphangiographic studies of a series of 107 patients in which the lower limbs were affected. Br J Surg 1957; 45(189):1-9
4 Koehler PR. Complications of lymphography. Lymphology 1968; $1(4): 116-120$

5 Vaughan BF. CT of swollen legs. Clin Radiol 1990;41(1):24-30

6 Suresh S, Kumaraswami V, Suresh I, et al. Ultrasonographic diagnosis of subclinical filariasis. J Ultrasound Med 1997;16(1): 45-49

7 Weissleder H, Weissleder R. Lymphedema: evaluation of qualitative and quantitative lymphoscintigraphy in 238 patients. Radiology 1988;167(3):729-735

8 Case TC, Witte CL, Witte MH, Unger EC, Williams WH. Magnetic resonance imaging in human lymphedema: comparison with lymphangioscintigraphy. Magn Reson Imaging 1992;10(4): 549-558

9 Weissleder R, Elizondo G, Wittenberg J, Lee AS, Josephson L, Brady TJ. Ultrasmall superparamagnetic iron oxide: an intravenous contrast agent for assessing lymph nodes with MR imaging. Radiology 1990;175(2):494-498

10 Lohrmann C, Foeldi E, Speck O, Langer M. High-resolution MR lymphangiography in patients with primary and secondary lymphedema. AJR Am J Roentgenol 2006;187(2):556-561

11 Ferrucci JT. Advances in abdominal MR imaging. Radiographics 1998;18(6):1569-1586

12 Arrivé L, Azizi L, Lewin M, et al. MR lymphography of abdominal and retroperitoneal lymphatic vessels. AJR Am J Roentgenol 2007; 189(5):1051-1058

13 Arrivé L, Coudray C, Azizi L, et al. Pineapple juice as a negative oral contrast agent in magnetic resonance cholangiopancreatography [in French]. J Radiol 2007;88(11, Pt 1):1689-1694

14 El Mouhadi S, Arrivé L. Magnetic resonance lymphography of chyluria. Kidney Int 2010;78(7):712 